T. Arakawa and T. Ashikaga

Nagoya Math. J.

Vol. 175 (2004), 103-124

\title{
LOCAL SPLITTING FAMILIES OF HYPERELLIPTIC PENCILS, II
}

\author{
TATSUYA ARAKAWA AND TADASHI ASHIKAGA
}

\begin{abstract}
We propose certain obstructions for the existence of hyperelliptic splitting families of degenerations of curves. Moreover we determine the complete system of hyperelliptic atomic fibers of genus 3 .
\end{abstract}

\section{Introduction}

This paper is a continuation of [1]. We study splitting families of degenerations for hyperelliptic curves so that the original complicated singular fiber decomposes into several simple singular fibers by those local deformations.

The main aims of the present paper are the following.

Firstly we show that certain invariants for the singular fiber of a hyperelliptic pencil are conserved in the splitting families.

Secondly, we give a complete system $\mathcal{A}$ of hyperelliptic 'atomic fibers', i.e., the atoms of degenerations, of genus three.

The paper consists of 6 sections.

In $\S 1$, we show that an analytic splitting naturally induces a $C^{\infty}$ splitting in the usual sense of topology (cf. [7]). Namely, let $\left\{\varphi_{u}: S_{u} \rightarrow\right.$ $\bar{\Delta}\}_{u \in \Delta}$ be a splitting family of a degeneration $\varphi_{0}: S_{0} \rightarrow \bar{\Delta}$ of curves over the complex one-dimensional closed disk $\bar{\Delta}$. Then there exists a diffeomorphism $h: S_{0} \rightarrow S_{u}$ which fixes the fibers over the boundary $\partial \bar{\Delta}$. This is analogous to the $C^{\infty}$ local triviality of smooth analytic families of compact complex manifolds.

In $\S 2$, firstly we correct the definition of the type II singular fibers in hyperelliptic pencils, which we defined in [1] but was incomplete.

Secondly as an application of the existence of splitting families, we show in Proposition 2.6 that any fixed component of the relative canonical linear

Received August 13, 2001.

2000 Mathematics Subject Classification: Primary 14H15; Secondary 14J29, 14J17, $14 \mathrm{H} 45,30 \mathrm{~F} 10$. 
system of a germ of a hyperelliptic degeneration of genus 3 disappears via a finite times of local deformations.

In $\S 3$, we define a new invariant for the singular fiber of a hyperelliptic pencil, which we shall call the Namba number or $N$-number. We define it as the number of components of the classical link which appears in a certain natural way from the branch curve of the relative hyperelliptic involution of the degeneration.

In $\S 4$, firstly in Proposition 4.1, we show that the Horikawa index (cf. [1] §4) and the Euler contribution for the singular fiber of a hyperelliptic pencil are conserved in the splitting families. Our proof is based on the conservation of the local signature due to Endo [4] and Terasoma [14].

Secondly in Proposition 4.3, we show the similar conservation property of the N-number.

In $\S 5$, firstly in Proposition 5.1, we show that the conservation of the Horikawa index and the Euler contribution is not enough for the existence of a splitting family with the preassigned invariants. In the proof of this proposition, N-number plays a crucial role.

In the remaining part of $\S 5$ and in $\S 6$, we show that a complete system $\mathcal{A}$ of hyperelliptic 'atomic fibers' of genus three consists of the germs of types $0_{0}, I, I I(i)$ and $I I(i i i)$ defined in 2.2 (see Figure 1). More precisely, any germ $(\varphi, F)$ of a hyperelliptic degeneration of genus 3 splits into the members of $\mathcal{A}$ via several splitting families and equisingular deformations, and any member of $\mathcal{A}$ does not split furthermore. By $[1],(\varphi, F)$ splits stepwisely into some of germs of types $0_{0}, I, I I(i), I I(i i)$ and $I I(i i i)$. We show in Proposition 5.5 that any germ of type $I I(i i)$ splits into germs of types $I$ and $I I($ iii $)$ after an equisingular deformation. We construct this splitting family by taking a cyclic quotient of a certain splitting family of the stable reduction of the original degeneration. There is another way of the construction by using the double cover of a certain splitting family of a genus two fibration $(\S 5.6)$.

Acknowledgements. The authors thank Prof. Eiji Horikawa, Prof. Kazuhiro Konno, Prof. Miles Reid, Prof. Yukio Matsumoto, Dr. Shigeru Takamura and Dr. Mizuho Ishizaka for valuable discussions and helpful advices. Thanks are also due to Prof. Xiao Gang, Prof. Sampei Usui, Prof. Noboru Nakayama and Prof. Makoto Namba for useful advices. Thanks are also due to the referee for useful advices and comments. 


\section{$\S 1$. Analytic splitting and $C^{\infty}$ splitting}

In this section, we prove that an analytic splitting of a degeneration of curves naturally induces a $C^{\infty}$ splitting in the sense of Matsumoto [7].

1.1 Let $\Delta_{\epsilon}$ (resp. $\left.\bar{\Delta}_{\epsilon}\right)$ be the open (resp. closed) disk of radius $\epsilon$ with center at the origin on the complex plane $\mathbf{C}$. We denote by $\partial \bar{\Delta}_{\epsilon}$ the boundary of $\bar{\Delta}_{\epsilon}$. Let $S$ be a nonsingular complex surface and let $\varphi: S \rightarrow \Delta_{\epsilon}$ be a degeneration of curves of genus $g$ with a unique singular fiber (i.e. a fiber with singularities) $F=\varphi^{-1}(0)$. Let $M$ be a three-dimensional complex manifold and let $\Phi: M \rightarrow \Delta_{\epsilon} \times \Delta_{\delta}$ be a proper flat surjective holomorphic map such that $M_{u}=\Phi^{-1}\left(\Delta_{\epsilon} \times\{u\}\right)$ is a smooth surface for any $u \in \Delta_{\delta}$. We call $\Phi$ an analytic splitting family of $\varphi$ if the following conditions are satisfied:

(i) The restriction $\Phi_{0}: M_{0} \rightarrow \Delta_{\epsilon} \times\{0\}$ of $\Phi$ over $0 \in \Delta_{\delta}$ coincides with $\varphi$, (ii) For any $u \in \Delta_{\delta} \backslash\{0\}$, the restriction $\Phi_{u}: M_{u} \rightarrow \Delta_{\epsilon} \times\{u\}$ has $l$ singular fibers where $l$ is an integer greater than or equal to 1 and is independent on $u$.

Then we say that the fiber germ $[\varphi, F]$ splits into the fiber germs $\left[\Phi_{u}, F_{u, 1}\right], \cdots,\left[\Phi_{u}, F_{u, l}\right]$ via the splitting family $\Phi$, and sometimes write

$$
[\varphi, F] \longrightarrow\left[\Phi_{u}, F_{u, 1}\right]+\cdots+\left[\Phi_{u}, F_{u, l}\right]
$$

symbolically. If $l \geq 2$, then we call $\Phi$ an analytic proper splitting family of $\varphi$. If $l=1$, then we call $\Phi$ an equisingular deformation of $\varphi$.

Proposition 1.2. Let $\Phi: M \rightarrow \Delta_{\epsilon} \times \Delta_{\delta}$ be an analytic splitting family of a degeneration $\varphi: S \rightarrow \Delta_{\epsilon}$. Then there exist real numbers $\epsilon^{\prime}, \delta^{\prime}$ $\left(0<\epsilon^{\prime}<\epsilon, 0<\delta^{\prime}<\delta\right)$ such that the restriction $\bar{\Phi}: \bar{M} \rightarrow \bar{\Delta}_{\epsilon^{\prime}} \times \bar{\Delta}_{\delta^{\prime}}$ of $\Phi$ to $\bar{M}=\Phi^{-1}\left(\bar{\Delta}_{\epsilon^{\prime}} \times \bar{\Delta}_{\delta^{\prime}}\right)$ has the following properties:

(a) Let $\mathcal{D}$ be the discriminant locus of $\Phi$ in $\Delta_{\epsilon} \times \Delta_{\delta}$. Then $\mathcal{D}$ does not intersect $\partial \bar{\Delta}_{\epsilon^{\prime}} \times \bar{\Delta}_{\delta^{\prime}}$.

(b) Put $\bar{M}_{u}=\bar{\Phi}^{-1}\left(\bar{\Delta}_{\epsilon^{\prime}} \times\{u\}\right)$ and $\partial \bar{M}_{u}=\bar{\Phi}^{-1}\left(\partial \bar{\Delta}_{\epsilon^{\prime}} \times\{u\}\right)$ for any $u \in \bar{\Delta}_{\delta^{\prime}}$. Let $\bar{\Phi}_{u}: \bar{M}_{u} \rightarrow \bar{\Delta}_{\epsilon^{\prime}} \times\{u\}$ be the restriction of $\bar{\Phi}$ over $u \in \bar{\Delta}_{\delta^{\prime}}$. Then there exists a diffeomorphism

$$
f_{u}: \bar{M}_{0} \longrightarrow \bar{M}_{u}
$$


with $f_{u}\left(\partial \bar{M}_{0}\right)=\partial \bar{M}_{u}$ so that the composition map $\left.\mathbf{i}_{u} \circ \bar{\Phi}_{u} \circ f_{u}\right|_{\partial \bar{M}_{0}}$ coincides with the restriction map $\left.\Phi_{0}\right|_{\partial \bar{M}_{0}}$, where $\mathbf{i}_{u}: \bar{\Delta}_{\epsilon^{\prime}} \times\{u\} \rightarrow$ $\bar{\Delta}_{\epsilon^{\prime}} \times\{0\}$ is the natural map induced by the identity of the first factor.

Namely, an analytic splitting family $\Phi$ induces a $C^{\infty}$ splitting of $\bar{M}_{0}$ to $\bar{M}_{u}$ in the sense of [7, Definition 3.4].

Proof. We fix complex coordinates $t$ and $u$ on $\Delta_{\epsilon}$ and $\Delta_{\delta}$, respectively. Denote by $p r_{1}: \Delta_{\epsilon} \times \Delta_{\delta} \rightarrow \Delta_{\epsilon}$ and $p r_{2}: \Delta_{\epsilon} \times \Delta_{\delta} \rightarrow \Delta_{\delta}$ the natural projections. There exist real numbers $\epsilon^{\prime}$ and $\delta^{\prime}\left(0<\epsilon^{\prime}<\epsilon, 0<\delta^{\prime}<\delta\right)$, an open covering $M=\left(\cup_{i \in \mathbf{I}} U_{i}\right) \cup\left(\cup_{j \in \mathbf{J}} U_{j}\right)$ (for $\mathbf{I} \cap \mathbf{J}=\emptyset$ ) and a system of coordinates $\left(z_{k}^{(1)}, z_{k}^{(2)}, u_{k}\right)$ on $U_{k}(k \in \mathbf{I} \cup \mathbf{J})$ which satisfy the following:

(i) I is a finite set and $\Phi^{-1}\left(\partial \bar{\Delta}_{\epsilon^{\prime}} \times\{0\}\right)$ is contained in $\cup_{i \in \mathbf{I}} U_{i}$. Moreover the set $\Phi\left(\cup_{i \in \mathbf{I}} U_{i}\right) \cap \mathcal{D}$ is empty.

(ii) For sufficiently small real numbers $\epsilon^{\prime \prime}$ and $\delta^{\prime \prime}\left(0<\epsilon^{\prime \prime}<\epsilon^{\prime}, 0<\delta^{\prime \prime}<\delta^{\prime}\right)$, we put the region $\mathcal{N}$ on $\Delta_{\epsilon} \times \Delta_{\delta}$ by

$$
\mathcal{N}=\left\{t \in \Delta_{\epsilon}\left|\epsilon^{\prime}-\epsilon^{\prime \prime} \leq\right| t \mid \leq \epsilon^{\prime}+\epsilon^{\prime \prime}\right\} \times\left\{u \in \Delta_{\delta}|| u \mid \leq \delta^{\prime \prime}\right\} .
$$

Then the set $\Phi\left(\cup_{j \in \mathbf{J}} U_{j}\right) \cap \mathcal{N}$ is empty.

(iii) For $k \in \mathbf{I} \cup \mathbf{J}$, we have $u_{k}=u$. The map $p r_{2} \circ \Phi$ on $U_{k}$ coincides with the third projection $\left(z_{k}^{(1)}, z_{k}^{(2)}, u_{k}\right) \rightarrow u_{k}$.

(iv) For $i \in \mathbf{I}$, we have $z_{i}^{(2)}=t$. The map $p r_{1} \circ \Phi$ on $U_{i}$ coincides with the second projection $\left(z_{i}^{(1)}, z_{i}^{(2)}, u_{i}\right) \rightarrow z_{i}^{(2)}$.

The transition function on $U_{k} \cap U_{l}(k, l \in \mathbf{I} \cup \mathbf{J})$ is given by $z_{k}^{(\alpha)}=$ $f_{k l}^{(\alpha)}\left(z_{l}^{(1)}, z_{l}^{(2)}, u_{l}\right)(\alpha=1,2)$ and $u_{k}=u_{l}$, where $f_{k l}^{(\alpha)}\left(z_{l}^{(1)}, z_{l}^{(2)}, u_{l}\right)$ is a holomorphic function of variables $z_{l}^{(1)}, z_{l}^{(2)}$ and $u_{l}$. Moreover, if $k, l \in \mathbf{I}$, then we have $f_{k l}^{(2)}\left(z_{l}^{(1)}, z_{l}^{(2)}, u_{l}\right)=z_{l}^{(2)}$. Now let $\left\{\mu_{k}\right\}_{k \in \mathbf{I} \cup \mathbf{J}}$ be the partition of unity

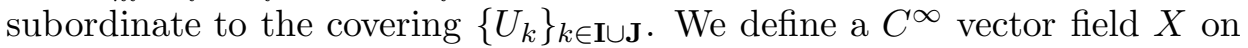
$M$ by

$$
\sum_{k \in \mathbf{I} \cup \mathbf{J}} \mu_{k} \frac{\partial}{\partial u_{k}}
$$

Then $X$ is written on $U_{k}(k \in \mathbf{I} \cup \mathbf{J})$ as

$$
\sum_{\alpha=1}^{2}\left(\sum_{l} \mu_{l} \frac{\partial f_{k l}^{(\alpha)}}{\partial u_{k}}\right) \frac{\partial}{\partial z_{k}^{(\alpha)}}+\frac{\partial}{\partial u_{k}}
$$


Moreover, on $U_{i} \cap \Phi^{-1}(N)(i \in \mathbf{I}), X$ is written as

$$
\left(\sum_{l} \mu_{l} \frac{\partial f_{k l}^{(1)}}{\partial u_{k}}\right) \frac{\partial}{\partial z_{k}^{(1)}}+\frac{\partial}{\partial u_{k}}
$$

By changing $\delta$ sufficiently small if necessary, it follows from the fundamental theorem of ordinary differential equation that there exists the integral curve with respect to $X$. Namely, there exists a diffeomorphism

$$
f: M_{0} \times \Delta_{\delta} \longrightarrow M .
$$

By (1.2.1) and (1.2.2), $f$ naturally induces the desired diffeomorphism.

Q.E.D.

\section{$\S 2$. Special classes of fiber germs and relative canonical system}

2.1 Let $\varphi: S \rightarrow \Delta_{\epsilon}$ be a hyperelliptic degeneration of curves of genus $g$ with a unique singular fiber $F=\varphi^{-1}(0)$. In [1] $\S 1$, we defined special classes type $0_{0}$, class I and class II of singular fiber germs of hyperelliptic degenerations. However the definition of class II were incomplete there. Here we correct it in the following way.

Let $B$ be a branch curve on $W=\mathbf{P}^{1} \times \Delta_{\epsilon}$ corresponding to the double cover of $W$ which is birationally equivalent to the relative canonical map of $\varphi$. $B$ is determined up to elementary transformations of the ambient $\mathbf{P}^{1}$ bundle $W$. Let $\psi: W \rightarrow \Delta_{\epsilon}$ be the natural projection and $\Gamma_{0}$ the fiber of $\psi$ over $0 \in \Delta_{\epsilon}$. If $B$ contains $\Gamma_{0}$, then we set $B_{h o r}:=B-\Gamma_{0}$ and otherwise we set $B_{h o r}=B$, which we call the horizontal part of $B$.

The branch curve $B$ is said to be trivial if one of the following two conditions is satisfied:

(a) $B_{\text {hor }}$ intersects $\Gamma_{0}$ at just one point $Q_{1}$ and $B_{\text {hor }}$ is not tangential to $\Gamma_{0}$ at $Q_{1}$, i.e. for the blow-up at $Q_{1}$, the proper transform of $\Gamma_{0}$ and that of $B_{\text {hor }}$ do not intersect.

(b) $B_{\text {hor }}$ intersects $\Gamma_{0}$ at just two points $Q_{1}$ and $Q_{2}$ such that $B_{\text {hor }}$ is smooth at $Q_{1}$ meeting transversally to $\Gamma_{0}$ and $B_{\text {hor }}$ is not tangential to $\Gamma_{0}$ at $Q_{2}$.

A branch curve which is not trivial is called nontrivial. Note that any trivial branch curve is modified to nontrivial one via a finite times of elementary transformations and vice versa. 
Now we say the $[\varphi, F]$ belongs to class II if at least one nontrivial branch curve $B$ contains $\Gamma_{0}$ and moreover any nontrivial branch curve $B$ with $B \supset \Gamma_{0}$ have the five properties (a) through (e) in $\$ 1.3 .3$ of [1].

Note that the definition in [1] lacks the non-triviality condition.

2.2 Let us recall the classification [1] of the fiber germs of hyperelliptic degenerations of genus three which belong to these special classes (See Figure 1):

(1) $[\varphi, F]$ is of type $0_{0}$ if and only if we can choose a branch curve $B$ which is smooth and meets $\Gamma_{0}$ transversally except at one point $P$ where the order of contact is two. In this class, the singular fiber $F$ is an irreducible stable curve with one node.

(2) $[\varphi, F]$ belongs to class I if and only if we can choose a branch curve $B$ which does not contain $\Gamma_{0}$ and meets $\Gamma_{0}$ transversally except at one point $P$ which is an ordinary singularity of $B$ of multiplicity 4 . In this class, the singular fiber $F$ is a stable curve with two nodes consisting of two smooth elliptic components.

(3) Class II consists of three subclasses II(i), II(ii) and II(iii). In any cases we can choose a branch curve $B$ such that $B$ contains $\Gamma_{0}$ and $B_{h o r}$ has the following properties, respectively:

(II(i)) $B_{\text {hor }}$ meets $\Gamma_{0}$ at two points $P$ and $Q$, which are ordinary singularities of $B$ of multiplicity 4 and 6 , respectively. Then $F$ is a stable curve with one node consisting of two smooth components of genera one and two respectively.

(II(ii)) $B_{\text {hor }}$ meets $\Gamma_{0}$ at two points $P$ and $Q$ and both of them are as follows: $B$ has three local irreducible components which are smooth and mutually tangential of order two. (Note that one of them is $\Gamma_{0}$.) Then $F$ has an irreducible decomposition $2 F^{\prime}+2 F^{\prime \prime}+2 F^{\prime \prime \prime}$ where $F^{\prime}, F^{\prime \prime}$ and $F^{\prime \prime \prime}$ are nonsingular curves of genera one, zero and one respectively, and the intersection numbers are $\left(F^{\prime}\right)^{2}=\left(F^{\prime \prime \prime}\right)^{2}=-1,\left(F^{\prime \prime}\right)^{2}=-2$, $F^{\prime} F^{\prime \prime}=F^{\prime \prime} F^{\prime \prime \prime}=1$ and $F^{\prime} F^{\prime \prime \prime}=0$.

(II(iii)) $B_{\text {hor }}$ meets $\Gamma_{0}$ at one point $P . B$ has five local irreducible components at $P$ which are smooth and mutually tangential of order two. (Note that one of them is $\Gamma_{0}$.) Then $F$ is written as $F=2 F^{\prime}$ where $F^{\prime}$ is a nonsingular curve of genus two. 

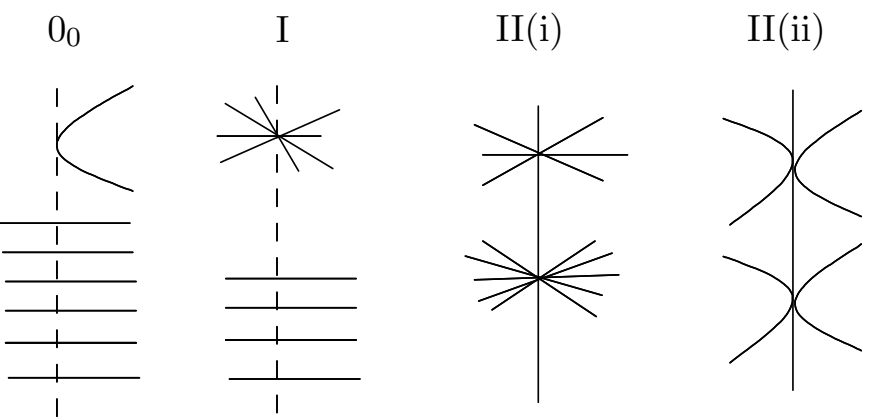

\section{II(iii)}
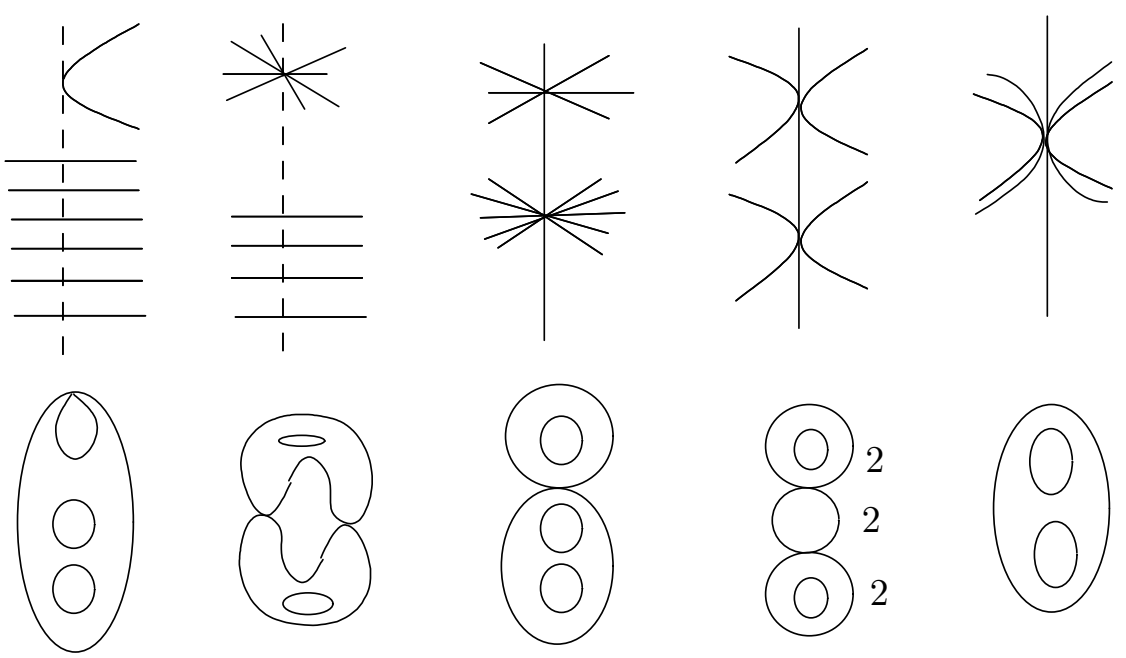

(Figure 1)

2.3 Horikawa [6] gives a method to determine relative (pluri)canonical systems of fiber germs of hyperelliptic degenerations explicitly. Here we will give a brief sketch of this method for class II (i) fiber germs of hyperelliptic degenerations of genus three.

Let $B$ be a branch curve on $W$ with the properties in 2.2 (II(i)). Then a canonical resolution of $B$ is given by the minimal embedded resolution $\sigma=\sigma_{2} \sigma_{1}: W_{2} \rightarrow W$ of $B$ where $\sigma_{i}$ is a blow-up $(i=1,2)$. The exceptional set of $\sigma$ is written as $D_{1}+D_{2}$ where $D_{1}, D_{2}$ are nonsingular rational curves on $W_{2}$. Let $B_{2}$ denote the proper transform of $B$ on $W_{2}$ and $S_{2}$ the double cover of $W_{2}$ whose branch curve is $B_{2}$. Then a holomorphic 2-form on $S_{2}$ is the pull back of a section of the bundle $K_{W_{2}}+L_{2}$ on $W_{2}$ where $L_{2}$ is a square-root bundle of $B_{2}$. Let $H$ denote a section of $\psi: W \rightarrow \Delta_{\epsilon}$. Since $B_{2}=\sigma^{*} B-4 D_{1}-6 D_{2}, K_{W_{2}} \cong \sigma^{*} K_{W}+D_{1}+D_{2}, B \cong 8 H$ and $K_{W} \cong-2 H$, we have $K_{W_{2}}+L_{2} \cong \sigma^{*}(2 H)-D_{1}-2 D_{2}$. Consequently we get the following identification:

$$
\Gamma\left(K_{S_{2}}\right) \cong\left\{\psi \in \Gamma(2 H) ; \sigma^{*}(\psi) \geq-D_{1}-2 D_{2}\right\}
$$

Let $(x: y, t)$ be coordinates of $W=\mathbf{P}^{1} \times \Delta_{\epsilon}$ such that $\sigma^{*}(x) \geq D_{1}$ and $\sigma^{*}(y) \geq D_{2}$. Since $\sigma^{*}(t) \geq D_{1}+D_{2}$, we conclude that the right hand side of (2.3.1) is generated by $t^{2} x^{2}, t x y$ and $t y^{2}$ over $\mathbf{C}\{t\}$. 
Moreover, since the identification (2.3.1) is given by $(\psi) \mapsto \sigma^{*}(\psi)-$ $D_{1}-2 D_{2}$, we also have $\mathrm{Bs}\left|K_{S_{2}}\right|=2 E$ where $E$ is the reduced part of the pull back of the proper transform of $\Gamma_{0} \subset W$.

Since $S_{2}$ is given by a blow-up of $S$ whose exceptional curve is $E$, we finally conclude that the center of this blow-up, which is nothing but the node of the singular fiber, is the unique base point of the (relative) canonical system.

Applying the same arguments for fiber germs in other classes, we have:

LEMMA 2.4. With an appropriate choice of the coordinates $(x: y, t)$ of $W$, the relative canonical system of a fiber germs $[\varphi, F]$ of a hyperelliptic degeneration of genus three is generated by $\varphi_{0}, \varphi_{1}, \varphi_{2}$ over $\mathbf{C}\{t\}$ where

$$
\begin{aligned}
& \left(\varphi_{0}, \varphi_{1}, \varphi_{2}\right)
\end{aligned}
$$

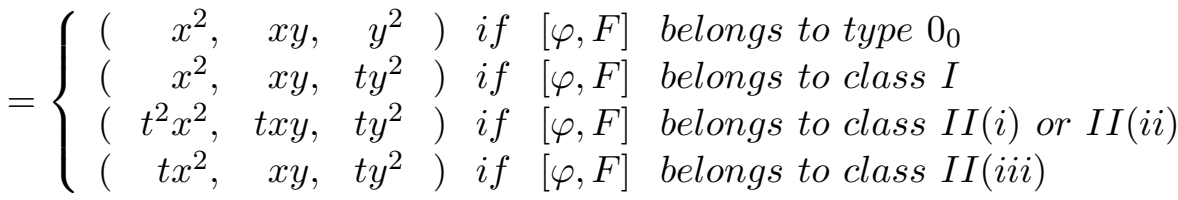

COROLlARY 2.5. The relative canonical system of a singular fiber germ of genus three is fixed point free if the germ belongs to type $0_{0}$, class $I$ or class II. If the germ belongs to type $0_{0}$ or class $I$, the relative canonical system has no base point either.

Note that the first half of Corollary 2.5 is well known by the general theory (cf. [10]).

By [1], any germ of a hyperelliptic degeneration of genus 3 splits into the set of germs in $0_{0}, I, I I(i), I I(i i)$ and $I I(i i i)$ via a finite times of hyperelliptic splitting families. Hence we have the following:

Proposition 2.6. Any fixed component of the relative canonical system of a germ of a hyperelliptic degeneration of genus three disappears via a finite times of local deformations.

Remark 2.7. If we choose alternative branch curves for a fiber germ, we may obtain other generators of the canonical system. In fact, if we apply the above method to the proper transform $B^{\prime}$ of the branch curve $B$ in $\S 2.3$ by the elementary transformation $W \rightarrow W$ whose center is the 6-ple point of $B$, we get generators $\left(x^{2}, x y, t y^{2}\right)$ instead of $\left(t^{2} x^{2}, t x y, t y^{2}\right)$ in Lemma 2.4 (with an appropriate choice of coordinate systems) . 


\section{§3. A monodromy invariant}

In this section, we define a certain monodromy invariant of a fiber germ of a hyperelliptic degeneration and calculate it for the fiber germs of genus three which belong to the special classes defined in $\S 2.2$.

3.1 We use the same notation as in $\S 2.1$. Let $\gamma \subset \Delta_{\epsilon}$ be a simple closed path winding around the origin $0 \in \Delta_{\epsilon}$ once. Then the restriction of $\psi$ on Supp $B$ induces an unramified covering of $\gamma$. Moreover $\psi^{-1}(\gamma) \cap$ Supp $B$ is contained in $\Delta_{N} \times \gamma \subset W$, where $\Delta_{N}$ is a disk in $\mathbf{P}^{1}$ of sufficiently large radius. Since $\Delta_{N} \times \gamma$ is canonically embedded in a three-dimensional sphere, we may consider $\psi^{-1}(\gamma) \cap \operatorname{Supp} B$ as a classical link.

Let $\mathcal{N}(B)$ denote the number of components of the link $\psi^{-1}(\gamma) \cap$ Supp $B$. Note that $\mathcal{N}(B)$ does not depend on the choice of the closed path $\gamma$. Furthermore:

Proposition-Definition 3.2. $\quad \mathcal{N}(B)$ does not depend on the choice of the branch curve $B$. We denote it by $\mathcal{N}([\varphi, F])$, and call it the $N$-number (Namba number) of the germ $[\varphi, F]$.

First proof of Proposition 3.2. Let $B$ and $B^{\prime}$ be two branch curves on $W$ corresponding to $\varphi$. If we restrict $\varphi$ over a neighborhood of $\gamma$, then the relative canonical map of $\varphi$ becomes a morphism. Let $R$ be the ramification divisor of this morphism. Then both $\operatorname{Supp} B$ and Supp $B^{\prime}$ are isomorphic to Supp $R$ over there. Therefore $\psi^{-1}(\gamma) \cap \operatorname{Supp} B$ is homeomorphic to $\psi^{-1}(\gamma) \cap \operatorname{Supp} B^{\prime}$. Hence $\mathcal{N}(B)=\mathcal{N}\left(B^{\prime}\right)$. (Remark that $\psi^{-1}(\gamma) \cap \operatorname{Supp} B$ and $\psi^{-1}(\gamma) \cap \operatorname{Supp} B^{\prime}$ are not necessarily equivalent to each other as links.)

Q.E.D

3.3 In order to give an alternative proof of Proposition 3.2, let us recall the notion of the braid monodromy of branched coverings (cf. [12]). Let $U \subset \Delta_{\epsilon}$ be an open neighborhood of $\gamma$ such that the branch curve $B$ restricted over $U$ is an unbranched covering of $U$ of degree $n=2 g+2$. Hence we have a natural homomorphism

$$
\Psi_{B}: \pi_{1}(U, p) \rightarrow \mathbf{B}_{n}
$$

where $p \in \gamma$ is the base point and $\mathbf{B}_{n}$ is the braid group of $n$-strings. We call $\Psi_{B}$ the braid monodromy of the branch curve $B$. Note that $\Psi_{B}(\gamma)$ gives a braid representation of the link $\psi^{-1}(\gamma) \cap \operatorname{Supp} B$. 
Now let $\tau: W \rightarrow W$ be an elementary transformation and let $B_{1}$ be the transformation of $B$ by $\tau$ as the branch curve (cf. [5], [6]). Then we have:

LEMmA 3.4. The braid $\Psi_{B_{1}}(\gamma) \in \mathbf{B}_{n}$ is given as a transform of $\Psi_{B}(\gamma)$ $\in \mathbf{B}_{n}$ by a full Dehn twist, and hence $\mathcal{N}(B)=\mathcal{N}\left(B_{1}\right)(=: \mathcal{N}([\varphi, F])$.

Proof. Let $x$ and $t$ be a local coordinate of the fiber and of $\Delta_{\epsilon}$, respectively. Then the elementary transformation $\tau: W \rightarrow W$ is given by $(x, t) \mapsto$ $(t x, t)$. On the other hand, suppose that $\gamma$ is given by $\theta \mapsto t=\exp (2 \pi \sqrt{-1} \theta)$ where $\theta \in[0,1]$ is a parameter. Then $\tau$ is given by $x \rightarrow \exp (2 \pi \sqrt{-1} \theta) \cdot x$ over a point $t(\theta)$ of $\gamma$. This implies the assertion.

Proposition 3.2 follows from Lemma 3.4, since every two branch curves $B$ and $B^{\prime}$ on $W$ corresponding to the same degeneration $\varphi$ are transformed to each other via a finite number of elementary transformations.

The following characterizations are useful to calculate $N$-numbers of given hyperelliptic degenerations:

LEMMA 3.5. (i) $\mathcal{N}([\varphi, F])$ coincides with the number of local irreducible components of $B$ which are not vertical.

(ii) $\mathcal{N}([\varphi, F])$ coincides with the number of cyclic permutations in the minimal decomposition of the image of $\gamma \in \pi_{1}(U, p)$ in the symmetric group $S_{n}$

Proof. The assertion (i) is clear by the definition, while (ii) follows from (i) since each of the local irreducible components of $B$ which are not vertical induces one of the cyclic permutations appearing in the minimal decomposition of the image of $\gamma \in \pi_{1}(U, p)$.

The following is a direct consequence of Lemma 3.5 (i):

LEMMA 3.6. Let $[\varphi, F]$ be a fiber germ of hyperelliptic degenerations of genus three which belongs to either Type $0_{0}$, Class I or Class II. Then

$$
\mathcal{N}([\varphi, F])=\left\{\begin{array}{lllll}
7 & \text { if } & {[\varphi, F]} & \text { belongs to } & 0_{0} \\
8 & \text { if } & {[\varphi, F]} & \text { belongs to } & \mathrm{I} \text { or } \mathrm{II}(\mathrm{i}) \\
4 & \text { if } & {[\varphi, F]} & \text { belongs to } & \mathrm{II}(\mathrm{ii}) \text { or } \mathrm{II}(\mathrm{iii})
\end{array}\right.
$$




\section{$\S 4$. Conservations of invariants}

Let $\varphi: S \rightarrow \Delta_{\epsilon}$ be a hyperelliptic degeneration of curves of genus $g$ with a unique singular fiber $F=\varphi^{-1}(0)$. Let $\mathcal{E}(F), \mathcal{H}([\varphi, F])$ and $\mathcal{N}([\varphi, F])$ be the Euler contribution, the Horikawa index (cf. [1] §4) and the N-number respectively. Let $\Phi: M \rightarrow \Delta_{\epsilon} \times \Delta_{\delta}$ be a hyperelliptic splitting family of $\varphi$, i.e. $\Phi$ is an analytic splitting family of $\varphi$ such that the general fiber of $\Phi$ is a hyperelliptic curve. In this section, we discuss the conservation properties of the above three invariants under $\Phi$.

Proposition 4.1. Assume that the germ $[\varphi, F]$ splits into the germs $\left[\Phi_{u}, F_{u, 1}\right], \cdots,\left[\Phi_{u}, F_{u, l}\right]$ via a hyperelliptic splitting family $\Phi$. Then :

$$
\mathcal{E}(F)=\sum_{i=1}^{l} \mathcal{E}\left(F_{u, i}\right), \quad \mathcal{H}([\varphi, F])=\sum_{i=1}^{l} \mathcal{H}\left(\left[\Phi_{u}, F_{u, i}\right]\right)
$$

for any $u \in \Delta_{\delta}$.

Proof. By the argument of Terasoma [14], there exist a compact complex surface $V$ and a hyperelliptic holomorphic family $\mu: V \rightarrow \mathbf{P}^{1}$ of genus $g$ which satisfy the followings: Let $\left\{Q_{0}, Q_{1}, \ldots, Q_{n}\right\} \subset \mathbf{P}^{1}$ be the set of all critical values of $\mu$. Let $\Delta$ be a small disk neighborhood around $Q_{0}$ and let $\tilde{\mu}:=\left.\mu\right|_{\tilde{V}}: \tilde{V}=\mu^{-1}(\Delta) \rightarrow \Delta$ be the restriction map. Then:

(i) There exist orientation preserving diffeomorphisms $h: S \rightarrow \tilde{V}$ and $\alpha: \Delta_{\epsilon} \rightarrow \Delta$ such that $\tilde{\mu} \circ h=\alpha \circ \varphi$.

(ii) We have

$$
\sigma^{t o p}([\varphi, F])=\sigma^{t o p}\left(\left[\mu, \mu^{-1}\left(Q_{0}\right)\right]\right)
$$

where $\sigma^{\text {top }}$ is the topological local signature of the germ defined by Endo [4].

(iii) For any $1 \leq i \leq n, \mu^{-1}\left(Q_{i}\right)$ is a Lefschetz fiber.

Now we fix $u \in \Delta_{\delta} \backslash\{0\}$. After shrinking $\Delta_{\epsilon}$ if necessary, $\Phi_{u}: M_{u} \rightarrow$ $\Delta_{\epsilon} \times\{u\}$ is naturally diffeomorphic to $\varphi: S \rightarrow \Delta_{\epsilon}$ by Proposition 1.2 . Therefore from the condition (i), we can patch the two pieces $\left.\mu\right|_{V \backslash \tilde{V}}: V \backslash$ $\tilde{V} \rightarrow \mathbf{P}^{1} \backslash \Delta$ and $\Phi_{u}: M_{u} \rightarrow \Delta_{\epsilon} \times\{u\}$ along their boundaries, and obtain a locally analytic fibration (in the sense of [7] and [4]) $\mu^{\prime}: V^{\prime} \rightarrow \mathbf{P}^{1}$ such that: 
(a) $\mu^{\prime}$ is a hyperelliptic (differentiable) family which has just $l+n$ singular fibers. Each of them is isomorphic to each of $\left[\Phi_{u}, F_{u, 1}\right], \ldots,\left[\Phi_{u}, F_{u, l}\right]$, $\left[\mu, \mu^{-1}\left(Q_{1}\right)\right], \ldots,\left[\mu, \mu^{-1}\left(Q_{n}\right)\right]$ as holomorphic fiber germs.

(b) $V^{\prime}$ is naturally diffeomorphic to $V$.

Since the topological Euler number $\chi^{t o p}(V)$ of $V$ coincides with $\chi^{t o p}\left(V^{\prime}\right)$, the first assertion follows from the equalities

$$
\chi^{t o p}(V)=\mathcal{E}(F)+n-2(2 g-2), \quad \chi^{t o p}\left(V^{\prime}\right)=\sum_{i=1}^{l} \mathcal{E}\left(F_{u, i}\right)+n-2(2 g-2) .
$$

Now Endo's theorem [4] says that

$$
\begin{aligned}
& \operatorname{Sign}(V)=\sigma^{t o p}([\varphi, F])+\sum_{i=1}^{n} \sigma^{t o p}\left(\left[\mu, \mu^{-1}\left(Q_{i}\right)\right]\right), \\
& \operatorname{Sign}\left(V^{\prime}\right)=\sum_{i=1}^{l} \sigma^{t o p}\left(\left[\Phi_{u}, F_{u, i}\right]\right)+\sum_{i=1}^{n} \sigma^{t o p}\left(\left[\mu, \mu^{-1}\left(Q_{i}\right)\right]\right)
\end{aligned}
$$

where $\operatorname{Sign}(V)$ is the signature of the intersection form on $\mathrm{H}^{2}(V, \mathbf{R})$ and so on. Therefore we have

$$
\sigma^{t o p}([\varphi, F])=\sum_{i=1}^{l} \sigma^{t o p}\left(\left[\Phi_{u}, F_{u, i}\right]\right) .
$$

On the other hand, let $\sigma:=1 /(2 g+1) \cdot(g \mathcal{H}-(g+1) \mathcal{E})$ be the algebrogeometric local signature defined in [1, Definition 4.6]. Then $\sigma$ coincides with $\sigma^{t o p}$ by Terasoma's theorem [14]. Hence we have

$$
\sigma([\varphi, F])=\sum_{i=1}^{l} \sigma\left(\left[\Phi_{u}, F_{u, i}\right]\right) .
$$

Therefore from the first assertion and the definition of $\sigma$, the second assertion also follows.

Q.E.D.

4.2. Let $\gamma \subset \Delta_{\epsilon}$ be a closed path around the origin such that $\gamma \times \Delta_{\delta}$ does not intersect the discriminant locus on $\Delta_{\epsilon} \times \Delta_{\delta}$ with respect to $\Phi$. Then, there does not exist any singular fiber of $\Phi_{u}$ on the outside-region of $\gamma$ in $\Delta_{\epsilon} \times\{u\}$ for each $u \in \Delta_{\delta}$. Let $B_{u}$ be a branch curve on $W_{u}=\mathbf{P}^{1} \times \Delta_{\epsilon}$ with 
respect to the hyperelliptic degeneration $\Phi_{u}$ with several singular fibers, and let $\Psi_{u}: W_{u} \rightarrow \Delta_{\epsilon}$ be the natural projection. By the same way as in $\S 2$, the number $\mathcal{N}_{u}$ of components of the link $\Psi_{u}^{-1}(\gamma) \cap \operatorname{Supp} B_{u}$ is well-defined.

Proposition 4.3. Let $\Phi: M \rightarrow \Delta_{\epsilon} \times \Delta_{\delta}$ be a hyperelliptic splitting family of $\varphi$. Then we have $\mathcal{N}([\varphi, F])=\mathcal{N}_{u}$ for any $u \in \Delta_{\delta}$.

Proof. Let $\Phi_{K_{M / \Delta_{\epsilon} \times \Delta_{\delta}}}: M \rightarrow \mathcal{W}$ be the relative canonical rational map of $\Phi$. If we restrict $\Phi$ over a neighborhood of $\gamma \times \Delta_{\delta}$, then the ramification divisor $\mathcal{R}$ on $M$ with respect to $\Phi_{K_{M / \Delta_{\epsilon} \times \Delta_{\delta}}}$ is well-defined. Moreover $\Psi_{u}^{-1}(\gamma) \cap \operatorname{Supp} B_{u}$ is homeomorphic to $\Phi_{u}^{-1}(\gamma) \cap \operatorname{Supp} \mathcal{R}$. Since the restriction of $\operatorname{Supp} \mathcal{R}$ over $\gamma$ is unbranched covering of $\gamma$, Supp $\mathcal{R}$ is homeomorphic to the direct product $\left(\Phi_{0}^{-1}(\gamma) \cap \operatorname{Supp} \mathcal{R}\right) \times\left(\gamma \times \Delta_{\delta}\right)$ over $\gamma \times \Delta_{\delta}$. Therefore, for each $u \in \Delta_{\delta}, \Phi_{u}^{-1}(\gamma) \cap \operatorname{Supp} \mathcal{R}$ is homeomorphic to $\Phi_{0}^{-1}(\gamma) \cap \operatorname{Supp} B$, which we identify with $\varphi^{-1}(\gamma) \cap \operatorname{Supp} B$. Hence the assertion follows.

Q.E.D

4.4 Let $F_{u, j}=\Phi_{u}^{-1}\left(P_{u, j}\right)(j=1,2, \ldots, l)$ be all the singular fibers of $\Phi_{u}$ for $u \neq 0$. Then the braid monodromy $\Phi: \pi_{1}\left(\Delta_{\epsilon}-\left\{P_{u, 1}, \ldots P_{u, l}\right\}\right) \rightarrow \mathbf{B}_{n}$ is defined. In this situation, with an appropriate choice of branch curve, the image of the simple closed path $\gamma$ given in 4.2 by the braid monodromy of $[\varphi, F]$ is a product of the braid monodromies of $\left[\varphi_{1}, F_{u .1}\right], \ldots,\left[\varphi_{l}, F_{u . l}\right]$ in a suitable order. Therefore we have:

LEMMA 4.5.

$$
2 g+2-\mathcal{N}_{u} \leq \sum_{j=1}^{l}\left(2 g+2-\mathcal{N}\left(\left[\varphi_{j}, F_{u, j}\right]\right)\right)
$$

\section{$\S 5$. Existence and non-existence of certain splittings}

Let $\mathcal{J}$ be the set of fiber germs of hyperelliptic degenerations of genus 3 of types $0_{0}, I, I I(i), I I(i i)$ and $I I($ iii $)$ defined in 2.2. By Example 4.5 of [1] and easy calculations, the Horikawa indices and the Euler contributions of these types of germs are as follows:

\begin{tabular}{|c|c|c|c|c|c|}
\hline & $0_{0}$ & I & II (i) & II (ii) & II (iii) \\
\hline H-index & 0 & $2 / 3$ & $5 / 3$ & $10 / 3$ & $8 / 3$ \\
\hline E-cont. & 1 & 2 & 1 & 4 & 2 \\
\hline
\end{tabular}


Let $\Phi$ be a hyperelliptic proper splitting family $[\varphi, F] \rightarrow\left[\Phi_{u}, F_{u, 1}\right]+\cdots+$ $\left[\Phi_{u}, F_{u, l}\right]$ of a degeneration $\varphi$ such that all the germs $[\varphi, F],\left[\Phi_{u}, F_{u, 1}\right], \cdots$, $\left[\Phi_{u}, F_{u, l}\right]$ belong to $\mathcal{J}$. Proposition 4.1 implies that there are only two possibilities of this splitting as follows:

(a) $I I(i i) \rightarrow 0_{0}+0_{0}+I I(i)+I I(i)$,

(b) $I I(i i) \rightarrow I+I I(i i i)$.

We prove the non-existence of the family (a) and the existence of the family (b).

Proposition 5.1. There exists no hyperelliptic splitting family $[\varphi, F]$ $\rightarrow\left[\Phi_{u}, F_{u, 1}\right]+\left[\Phi_{u}, F_{u, 2}\right]+\left[\Phi_{u}, F_{u, 3}\right]+\left[\Phi_{u}, F_{u, 4}\right]$ such that the germs $[\varphi, F]$, $\left[\Phi_{u}, F_{u, 1}\right],\left[\Phi_{u}, F_{u, 2}\right],\left[\Phi_{u}, F_{u, 3}\right]$ and $\left[\Phi_{u}, F_{u, 4}\right]$ are of types $\operatorname{II}(i i), 0_{0}, 0_{0}, I I(i)$ and $I I(i)$, respectively.

Proof. Assume that such a splitting family $\Phi$ exists. Since $\mathcal{N}\left(\left[\Phi_{u}, F_{u, 1}\right]\right)$ $=\mathcal{N}\left(\left[\Phi_{u}, F_{u, 2}\right]\right)=7$ and $\mathcal{N}\left(\left[\Phi_{u}, F_{u, 3}\right]\right)=\mathcal{N}\left(\left[\Phi_{u}, F_{u, 4}\right]\right)=8$ by Lemma 3.6, it follows that $\mathcal{N}_{u} \geq 6$ by Lemma 4.5. Since $\mathcal{N}([\varphi, F])=4$, this contradicts Proposition 4.3.

Q.E.D.

COROllary 5.2. The conservation properties of the Horikawa indices and the Euler contributions described in Proposition 4.1 are not sufficient for the existence of hyperelliptic splitting families.

Remark 5.3. By a slight modification of the argument in Proposition 5.1 , we can show that a fiber germ of type $I I(i i)$ does not split into four germs as in (a) not only via one splitting family but also via stepwise several splitting families.

5.4 For the discussion of the family (b), we prepare some arguments of the monodromy. In general, let $\varphi: S \rightarrow \Delta_{\epsilon}$ be a degeneration of curves of genus $g \geq 2$ with a unique singular fiber $F=f^{-1}(0)$. If we fix $t_{0} \in \Delta^{*}$, then the monodromy action of $\pi_{1}\left(\Delta^{*}, t_{0}\right) \simeq \mathbf{Z}$ defines modulo isotopy an orientation-preserving homeomorphism $f_{\varphi}: S_{t_{0}} \rightarrow S_{t_{0}}$ of the smooth fiber $S_{t_{0}}$. Since the change of the base point $t_{0}$ corresponds to the conjugation in the mapping class group $\mathcal{M}_{g}$ of genus $g, \varphi$ induces a unique element $\left[f_{\varphi}\right] \in \widehat{\mathcal{M}}_{g}$ where $\widehat{\mathcal{M}}_{g}$ is the set of conjugacy classes of $\mathcal{M}_{g}$. We call $\left[f_{\varphi}\right]$ the topological monodromy of $\varphi$. Matsumoto-Montesinos' theorem [8] says that 
the element of $\widehat{\mathcal{M}}_{g}$ is realizable as a topological monodromy of a certain degeneration if and only if it is a conjugacy class of a pseudo-periodic map of negative twist. Moreover this class is completely determined by the invariants which are called valencies, screw numbers and the action of the extended partition graph ([13], [9]).

Now we consider fiber germs $[\varphi, F]$ of types $I, I I(i i)$ and $I I(i i i)$. We determine the topological monodromy of these fiber germs, and at the same time describe their semi-stable reductions explicitly.

If $[\varphi, F]$ is of type $I$, then $F$ itself is a stable curve and the topological monodromy is a full Dehn twist along the vanishing cycle which is a disjoint union of two simple closed curves.

Suppose $[\varphi, F]$ is of type $I I(i i)$. The branch curve $B$ on $W=\mathbf{P}^{1} \times \Delta_{\epsilon}$ corresponds to $\varphi$ is written by $\Gamma+\sum_{i=1}^{4} B_{i}$ where $\Gamma$ is a fiber of $\pi: W \rightarrow \Delta_{\epsilon}$, $\Gamma$ and $B_{i}(i=1,2)$ are mutually two-tangential at a point $P$ and $\Gamma$ and $B_{i}$ $(i=3,4)$ are mutually two-tangential at a point $Q(Q \neq P)$ (see Figure 2$)$.
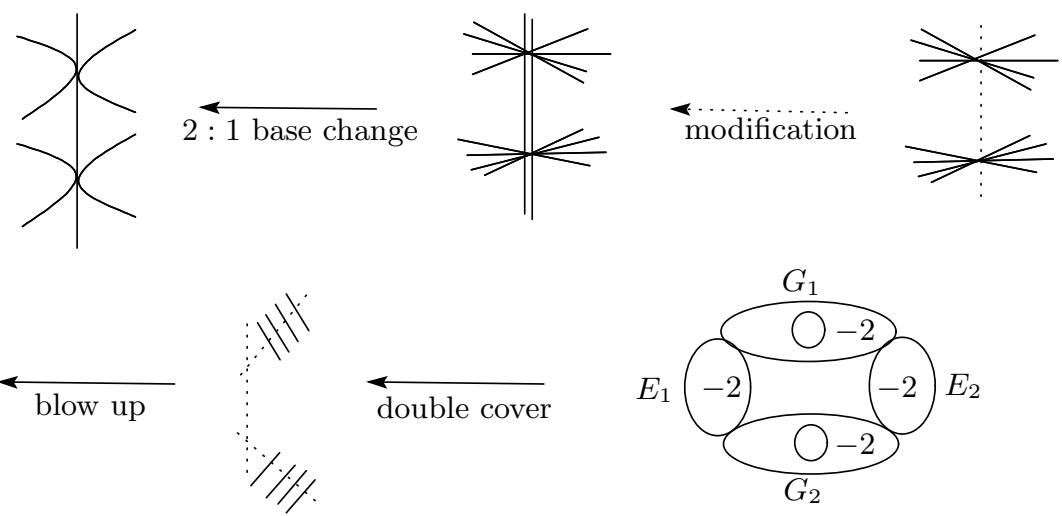

(Figure 2)

Let $\mu: \Delta_{\sqrt{\epsilon}} \rightarrow \Delta_{\epsilon}$ be the double cover with the branch point at the origin. Put $\widetilde{\mu}=i d_{\mathbf{P}^{1}} \times \mu: \widetilde{W}=\mathbf{P}^{1} \times \Delta_{\sqrt{\epsilon}} \rightarrow W$ where $i d_{\mathbf{P}^{1}}$ is the identity map of $\mathbf{P}^{1}$. The pull back $\widetilde{\mu}^{*} B$ is written by $2 \Gamma^{\prime}+\sum_{i=1}^{4} \sum_{j=1}^{2} B_{i j}^{\prime}$ where $\Gamma^{\prime}$ is a fiber of $\widetilde{\pi}: \widetilde{W} \rightarrow \Delta_{\sqrt{\epsilon}}, \Gamma^{\prime}$ and $B_{i j}^{\prime}(1 \leq i \leq 2,1 \leq j \leq 2)$ intersect transversally at a point $P^{\prime}$ and $\Gamma^{\prime}$ and $B_{i j}^{\prime}(3 \leq i \leq 4,1 \leq j \leq 2)$ intersect transversally at a point $Q^{\prime}\left(Q^{\prime} \neq P^{\prime}\right)$. Therefore the normalization of the 
fiber product of $S$ and $\Delta_{\sqrt{\epsilon}}$ over $\Delta_{\epsilon}$ coincides with the double cover $\widetilde{S}$ of $\widetilde{W}$ branched along $\sum_{i=1}^{4} \sum_{j=1}^{2} B_{i j}^{\prime}$. Let $S^{*}$ be the nonsingular model of $\widetilde{S}$ obtained by the canonical resolution $([5])$. Then the natural map

$$
\widetilde{\varphi}: S^{*} \rightarrow \Delta_{\sqrt{\epsilon}}
$$

has the singular fiber $\widetilde{F}=G_{1}+G_{2}+E_{1}+E_{2}$ where $G_{i}(i=1,2)$ are nonsingular elliptic curves, $E_{i}(i=1,2)$ are nonsingular rational curves, $G_{1}^{2}=G_{2}^{2}=E_{1}^{2}=E_{2}^{2}=-2, G_{i} E_{j}=1(1 \leq i, j \leq 2)$ and $G_{1} G_{2}=E_{1} E_{2}=0$ (see Figure 2). $\widetilde{\varphi}$ is the semi-stable reduction of $\varphi$.

On the other hand, there exists an automorphism $\rho: S^{*} \rightarrow S^{*}$ of order 2 compatible with the canonical involution of $\Delta_{\sqrt{\epsilon}}$ so that $\rho$ acts on $\widetilde{F}$ as follows: $\rho$ acts on $G_{i}(i=1,2)$ as an automorphism of order 2 without fixed points, $\rho\left(E_{1}\right)=E_{2}$ and $\rho\left(E_{2}\right)=E_{1}$. The quotient space of the fiber space $\widetilde{\varphi}$ by $\rho$ coincides with the original fiber space $\varphi$.

Therefore the topological monodromy of the degeneration $\varphi$ is isotopic to the pseudo-periodic map $f: \Sigma_{3} \rightarrow \Sigma_{3}$ of negative twist of a real two dimensional orientable closed manifold $\Sigma_{3}$ of genus 3 as follows: The admissible system $\mathcal{C}$ of cut curves consists of two disjoint simple closed (real) curves $C_{1}+C_{2}$ so that $\Sigma_{3}-\mathcal{C}$ consists of two connected components $\mathcal{B}_{1}, \mathcal{B}_{2}$ of genus 1 (see Figure 3 ). $f$ acts on $\mathcal{B}_{i}(i=1,2)$ as a periodic map of order 2 without fixed points, $f\left(C_{1}\right)=C_{2}, f\left(C_{2}\right)=C_{1}$ and $f^{2}$ acts on $\Sigma_{3}$ as the two-times Dehn twist along each of $C_{i}(i=1,2)$.

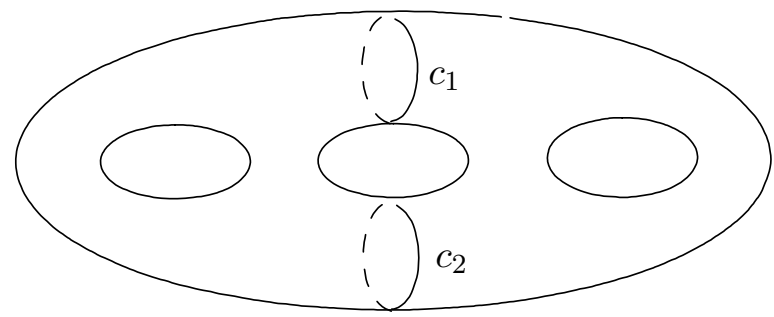

(Figure 3)

Suppose $[\varphi, F]$ is of type $I I(i i i)$. We get the stable family after a twoto-one base change. This is nothing but a smooth family of genus 3 . The topological monodromy is isotopic to a periodic map of order 2 without fixed points. 
Proposition 5.5. There exists a hyperelliptic splitting family $[\varphi, F] \rightarrow$ $\left[\Phi_{u}, F_{u, 1}\right]+\left[\Phi_{u}, F_{u, 2}\right]$ such that the germs $[\varphi, F],\left[\Phi_{u}, F_{u, 1}\right]$ and $\left[\Phi_{u}, F_{u, 2}\right]$ are of types II(ii), I and II(iii), respectively.

Proof. We fix the coordinates $t$ and $u$ on $\Delta_{\epsilon}$ and $\Delta_{\delta}$, respectively. Put $\boldsymbol{\Delta}:=\Delta_{\epsilon} \times \Delta_{\delta}$. We denote by $(x: y: z)$ a system of homogeneous coordinates of $\mathbf{P}^{2}$. Let $\mathcal{W}$ be the divisor on $\Delta_{\epsilon} \times \Delta_{\delta} \times \mathbf{P}^{2}$ defined by the equation

$$
\left(t^{2}-u^{2}\right)\left(x^{2}+y^{2}+z^{2}\right)+x y=0 .
$$

Let $\pi: \mathcal{W} \rightarrow \boldsymbol{\Delta}$ be the natural map and put $p r_{1}: \Delta \rightarrow \Delta_{\epsilon}$ and $p r_{2}$ : $\Delta \rightarrow \Delta_{\delta}$ the first and the second projection, respectively. We denote by $\mathcal{D}$ the discriminant locus of $\pi$. Since $\delta$ is sufficiently small, $\mathcal{D}$ is written by $D_{1}+D_{2}$ where $D_{1}=\{t+u=0\}$ and $D_{2}=\{t-u=0\}$. The fiber space $\pi$ has a conic bundle structure so that $\pi^{-1}(Q)(Q \in \mathcal{D})$ consists of two lines, which we denote by $L_{Q, 1}+L_{Q, 2}$.

We fix mutually distinct non-zero complex numbers $\alpha$ and $\beta$. Let $H_{i}$ $(1 \leq i \leq 4)$ be the divisors on $\boldsymbol{\Delta} \times \mathbf{P}^{2}$ defined by the equations $x+y+\alpha z=0$, $x+y+\beta z=0, x+y-\alpha z=0$ and $x+y-\beta z=0$, respectively. We set $B_{i}=H_{i} \cap \mathcal{W}(1 \leq i \leq 4)$ and put $B=\sum_{i=1}^{4} B_{i}$. Then $\pi^{-1}(Q) \cap B$ consists of mutually distinct eight points for any $Q \in \Delta$. Moreover if $Q \in \mathcal{D}$, then four points of $\pi^{-1}(Q) \cap B$ are on $L_{Q, 1}$ and the other four points are on $L_{Q, 2}$.

Let $\rho^{\prime}: \mathcal{V} \rightarrow \mathcal{W}$ be the double cover branched along $B . \mathcal{V}$ is smooth and is realized on $\boldsymbol{\Delta} \times \mathbf{P}^{2} \times \mathbf{C}$ by the equations (5.5.1) and

$$
\xi^{2}+(x+y+\alpha z)(x+y+\beta z)(x+y-\alpha z)(x+y-\beta z)=0,
$$

where $\xi$ is the coordinate of $\mathbf{C}$. We set

$$
\rho=\pi \circ \rho^{\prime}: \mathcal{V} \rightarrow \Delta .
$$

Then the general fiber $\rho^{-1}(Q)(Q \in \boldsymbol{\Delta} \backslash \mathcal{D})$ is a smooth hyperelliptic curve of genus 3, while the singular fiber $\rho^{-1}(Q)(Q \in \mathcal{D})$ consists of two nonsingular elliptic curves $G_{1}+G_{2}$ so that $G_{1}$ and $G_{2}$ meet transversally at two points $P_{1}$ and $P_{2}$.

Now we define the action $\bar{\tau}$ on $\boldsymbol{\Delta} \times \mathbf{P}^{2} \times \mathbf{C}$ by

$$
t \longmapsto-t, u \longmapsto u ; x \longmapsto-x, y \longmapsto-y, z \longmapsto z ; \xi \longmapsto-\xi .
$$

Then $\bar{\tau}$ preserves the divisor $\mathcal{V}$ and the restriction $\tau:=\bar{\tau} \mid \mathcal{V}$ induces an automorphism of $\mathcal{V}$ of order 2. The action $\tau$ is free from fixed points and has the following properties: 
(i) We denote by $Q_{u, j}\left(u \in \Delta_{\delta}, 1 \leq j \leq 2\right)$ the points on $\boldsymbol{\Delta}$ defined by $\mathrm{pr}_{2}^{-1}(u) \cap D_{j}$. Then $\tau$ maps the fiber $\pi^{-1}\left(Q_{u, 1}\right)$ isomorphically onto $\pi^{-1}\left(Q_{u, 2}\right)$.

(ii) If the point $Q$ on $\Delta$ is on the locus $p r_{1}^{-1}(0)$, then the restriction of $\tau$ to $\pi^{-1}(Q)$ induces an automorphism.

(iii) The restriction map of $\tau$ on the fiber $\pi^{-1}(\mathbf{0})$ over the origin $\mathbf{0} \in \boldsymbol{\Delta}$ induces the involution on each components $G_{1}$ and $G_{2}$, so that the map interchanges $P_{1}$ and $P_{2}$.

Let $M^{\prime}$ be the quotient smooth threefold of $\mathcal{V}$ by the group generated by $\tau$. We denote by $\Delta_{\epsilon^{2}}$ the quotient of $\Delta_{\epsilon}$ by the involution $t \longmapsto-t$. Then the natural map

$$
\Phi^{\prime}: M^{\prime} \longrightarrow \Delta_{\epsilon^{2}} \times \Delta_{\delta}
$$

induces a fiber space of hyperelliptic curves of genus 3. We denote by $\Phi_{u}^{\prime}: M_{u}^{\prime} \rightarrow \Delta_{\epsilon^{2}} \times\{u\}$ the restriction of $\Phi^{\prime}$ over $u \in \Delta_{\delta}$. Then:

(a) For $u \neq 0, M_{u}^{\prime}$ is smooth and the degeneration $\Phi_{u}^{\prime}$ has just two singular fibers so that the topological structures of these germs coincide with the topological structures of germs of type $I$ and $I I(i i i)$, respectively.

(b) The fiber of $\Phi_{0}^{\prime}$ is smooth except over 0 . The fiber $\left(\Phi_{0}^{\prime}\right)^{-1}(0)$ is the union of two multiple of two nonsingular elliptic curves $2 \widetilde{G_{1}}+2 \widetilde{G_{2}}$ so that $\widetilde{G_{1}}$ and $\widetilde{G_{2}}$ intersect at a point $\bar{P}$. Moreover $M_{0}$ has an $A_{1}$-singularity at $\bar{P}$.

After the two-to-one base change $\Delta_{\sqrt{\delta}} \rightarrow \Delta_{\delta}$, the $A_{1}$-singularity $\bar{P}$ is resolved by the method of Atiyah [3]. Considering the observation in $\S 4.4$, the resolution space becomes a desired splitting family.

Q.E.D.

Note that the germ of type $I I(i i)$ constructed in Proposition 5.5 has a very special analytic structure.

5.6 Here we give another construction of a splitting of a fiber germ in $I I(i i)$. This construction is simpler than the former, but the former seems more useful in the study of the monodromy types of singular fibers of the general members of the splitting families.

Let $t$ denote a coordinate on $\Delta_{\epsilon}$ and $(x: y)$ a homogeneous coordinate of $\mathbf{P}^{1}$. Let $B$ be the divisor on $W=\mathbf{P}^{1} \times \Delta_{\epsilon}$ defined by the equation

$$
t\left(x^{4}-t^{2} y^{4}\right)\left(t^{2} x^{4}-y^{4}\right)=0 .
$$


Let $\varphi: S \rightarrow \Delta_{\epsilon}$ be a hyperelliptic degeneration of genus three whose branch curve is $B$. Then the corresponding fiber germ $[\varphi, F]$ is of type $I I(i i)$ (cf. 2.2). Let $\sigma: W \rightarrow W$ be the action given by

$$
x \longmapsto-x, y \longmapsto y, t \longmapsto t .
$$

Then $\sigma$ preserves the divisor $B$ and is lifted to $S$ as a fiber-preserving holomorphic involution in two ways, namely if one is $\tilde{\sigma}: S \rightarrow S$, then another is $\tilde{\sigma} h: S \rightarrow S$ where $h: S \rightarrow S$ is the hyperelliptic involution. It is easy to see that the fixed point locus of one of them, say $\tilde{\sigma}$, is $F^{\prime}+F^{\prime \prime \prime}$ while that of the other, say $\tilde{\sigma} h$, is $F^{\prime \prime}$ where $F^{\prime}, F^{\prime \prime}$ and $F^{\prime \prime \prime}$ are the same as in 2.2 (II (ii)).

Let $S \rightarrow T_{1}$ be the double covering induced by the involution $\tilde{\sigma}$. Then $T_{1}$ is a smooth surface and has a pencil of curves of genus two whose only singular fiber is of the form $\Gamma_{1}=\Gamma^{\prime}+2 \Gamma^{\prime \prime}+\Gamma^{\prime \prime \prime}$, where $\Gamma^{\prime}, \Gamma^{\prime \prime \prime}$ and $\Gamma^{\prime \prime \prime}$ are nonsingular curves of respective genera one, zero, one with intersection numbers $\Gamma^{\prime} \Gamma^{\prime \prime}=\Gamma^{\prime \prime} \Gamma^{\prime \prime \prime}=1, \Gamma^{\prime} \Gamma^{\prime \prime \prime}=0,\left(\Gamma^{\prime}\right)^{2}=\left(\Gamma^{\prime \prime \prime}\right)^{2}=-2$ and $\left(\Gamma^{\prime \prime}\right)^{2}=-1$. Moreover the branch locus on $T_{1}$ is $B_{T_{1}}=\Gamma^{\prime}+\Gamma^{\prime \prime \prime}$. Let $T_{1} \rightarrow T$ be the contraction of the $(-1)$ curve $\Gamma^{\prime \prime}$. Then naturally $T$ also has a pencil of curves of genus two and the branch locus $B_{T}$ on $T$ coincides with the unique singular fiber $\Gamma$ on $T$.

Let $B_{T, u}$ be the family of divisors on $T$ where $u \in \Delta_{\delta}$ is a parameter and $B_{T, u}$ is defined as the fiber of $T \rightarrow \Delta_{\epsilon}$ over the point $t=u \in \Delta_{\epsilon}$. Then since $B_{T, u}$ 's are smooth curves of genus two for $u \neq 0$ and $B_{T, 0}=B_{T}$ has an ordinary double point as the unique bad point, we get a splitting family $\Phi: M \rightarrow \Delta_{\epsilon} \times \Delta_{\delta}$ of genus 3 which satisfies (i) the degeneration $\Phi_{0}: M_{0} \rightarrow \Delta_{\epsilon} \times\{0\}$ coincides with $\varphi: S \rightarrow \Delta_{\epsilon}$ and (ii) the degeneration $\Phi_{u}: M_{u} \rightarrow \Delta_{\epsilon} \times\{u\}(u \neq 0)$ has two singular fibers $F_{u, 1}=\Phi_{u}^{-1}(0)$ and $F_{u, 2}=\Phi_{u}^{-1}(u)$.

Accola's result ([2] Lemma 5.10) says that a compact Riemann surface of genus three which is a double covering of a compact Riemann surface of genus two is hyperelliptic. Therefore the general fiber of $\Phi: M \rightarrow \Delta_{\epsilon} \times \Delta_{\delta}$ is hyperelliptic.

\section{§6. Hyperelliptic atomic fibers of genus 3}

Definition 6.1. The set of germs of fibers $\mathcal{A}=\left\{\left[\varphi_{i}, F_{i}\right]\right\}_{i \in I}$ of hyperelliptic degenerations of genus $g$ is said to be the complete system of hyperelliptic atomic fibers of genus $g$ if the following conditions are satisfied: 
(i) There exists no hyperelliptic proper splitting family of $\left[\varphi_{i}, F_{i}\right]$ for any $i \in I$.

(ii) The germ $[\varphi, F]$ of any hyperelliptic degeneration of genus $g$ splits into the members of $\mathcal{A}$ via a finite number of hyperelliptic splitting families.

More precisely, $[\varphi, F]$ has an equisingular deformation to $\left[\varphi^{\prime}, F^{\prime}\right]$ so that $\left[\varphi^{\prime}, F^{\prime}\right]$ splits into $\left[\Phi_{u}, F_{u, 1}\right], \cdots,\left[\Phi_{u}, F_{u, l}\right]$ via a certain hyperelliptic splitting family $\Phi: M \rightarrow \Delta_{\epsilon} \times \Delta_{\delta}$. If $\left[\Phi_{u}, F_{u, i}\right]$ for some $i(1 \leq i \leq l)$ is not contained in $\mathcal{A}$, then $\left[\Phi_{u}, F_{u, i}\right]$ has a hyperelliptic splitting family after an equisingular deformation (if it is necessary). We continue this process. Then after a finite steps, all the germs of singular fibers of resulting degenerations are contained in $\mathcal{A}$.

THEOREM 2. Let $\mathcal{A}$ be the set of germs of types $0_{0}, I, I I(i)$ and $I I($ iii $)$. Then $\mathcal{A}$ is the complete system of hyperelliptic atomic fibers of genus 3 .

Proof. By [1], any germ of a hyperelliptic degeneration of genus 3 splits into the set of germs of types $0_{0}, I, I I(i), I I(i i)$ and $I I(i i i)$ via a finite number of hyperelliptic proper splitting families (without using equisingular deformations).

Let $[\varphi, F]$ be any germ of type $I I(i i)$. Then $[\varphi, F]$ has an equisingular deformation to the special germ of type $I I(i i)$ which is constructed in Proposition 5.5. Indeed, the equation of a representative of the branch curve $B$ of $[\varphi, F]$ on $W=\mathbf{P}^{1} \times \Delta_{\epsilon}$ is written by

$$
t \prod_{i=1}^{2} \prod_{j=1}^{4}\left\{t+\left(x-a_{i}\right)^{2}\left(b_{i j}+g_{i j}(t)\right)+\left(x-a_{i}\right)^{3} f_{i j}(t, x)\right\}=0
$$

where $t$ is the coordinate of $\Delta, x$ is the inhomogeneous coordinate of $\mathbf{P}^{1}$, $a_{1}$ and $a_{2}$ are mutually distinct complex numbers, $b_{i 1}, \cdots, b_{i 4}(i=1,2)$ are mutually distinct non-zero complex numbers, $g_{i j}(t)$ and $f_{i j}(t, x)$ are holomorphic functions of $t$ and $(t, x)$ respectively. We define the divisor $\mathbf{B}$ on $\mathbf{W}=W \times \Delta_{\delta}$ by

$$
\begin{aligned}
t \prod_{i=1}^{2} \prod_{j=1}^{4}\{t+ & \left(x+(-1)^{i} u+a_{i} u-a_{i}\right)^{2}\left(\left(k_{i j}-b_{i j}\right) u+b_{i j}+(1-u) g_{i j}(t)\right) \\
& \left.+(1-u)\left(x+(-1)^{i} u+a_{i} u-a_{i}\right)^{3} f_{i j}(t, x)\right\}=0
\end{aligned}
$$


where $u$ is the coordinate of $\Delta_{\delta}$ and $k_{i j}(1 \leq i \leq 2,1 \leq j \leq 4)$ are complex numbers which satisfy $k_{11}=-k_{12}=k_{21}=-k_{22}, k_{13}=-k_{14}=k_{23}=-k_{24}$ and $k_{11} \neq k_{13}$. Then the resolution space of the double cover of $\mathbf{W}$ branched along $\mathbf{B}$ has the desired germ over $u=1$.

This germ splits into germs of types $I$ and $I I($ iii $)$ by Proposition 5.5. Since any germ of type $0_{0}, I, I I(i)$ or $I I(i i i)$ does not have proper splitting family by Proposition 4.1, the assertion follows.

Q.E.D.

Remark 6.3. By similar arguments as those in the proof of Theorem 6.2 , we can show that any singular fiber germ of type $I I(i i)$ has an equisingular deformation to the special germ which is constructed in 5.6.

\section{REFERENCES}

[1] T. Arakawa and T. Ashikaga, Local splitting families of hyperelliptic pencils I, Tôhoku Math. J., 53 (2001), 369-394.

[2] R. D.M. Accola, Topics in the Theory of Riemann Surface, Lecture Notes in Math., 1595 (1994), Splinger-Verlag, Berlin Heidelberg.

[3] M. F. Atiyah, On analytic surfaces with double points, Proc. Roy. Soc., A 247 (1958), 237-244.

[4] H. Endo, Meyer's signature cocycle and hyperelliptic fibrations, Math. Ann., 316 (2000), 237-257.

[5] E. Horikawa, On deformations of quintic surfaces, Invent. Math., 31 (1975), 43-85.

[6] _ On algebraic surfaces with pencils of curves of genus 2, In: Complex Analysis and Algebraic Geometry, a volume dedicated to K. Kodaira, pp. 79-90, Tokyo and Cambridge, Iwanami Shoten Publishers and Cambridge University Press, 1977.

[7] Y. Matsumoto,, Lefschetz fibrations of genus two - a topological approach, Proc. of the 37th Taniguchi symposium on topology and Teichmüller spaces held in Finland 1995, pp.123-148, World Scientific 1996.

[8] Y. Matsumoto and J. M. Montesinos-Amilibia, Pseudo-periodic maps and degeneration of Riemann surfaces I, II, Preprints, Univ. of Tokyo and Univ. Complutense de Madrid, 1991/1992.

[9] — Pseudo-periodic homeomorphisms and degeneration of Riemann surfaces, Bull. Amer. Math. Soc., 30 (1994), 70-75.

[10] M. Mendes Lopes, , The relative canonical algebra for genus 3 fibrations, Thesis, Univ. of Warwick 1988.

[11] W. Meyer, Die Signatur von Flächenbündeln, Math. Ann., 201 (1973), 239-264.

[12] M. Namba, Branched Coverings and Algebraic Functions, Research Notes in Math. 161, Pitman-Longman, 1987.

[13] J. Nielsen, Surface transformation classes of algebraically finite type, Mat.-Fys. Medd. Danske Vid. Selsk., 21 1944, 3-89. English translation: in Collected Papers 2, Birkhäuser, 1986. 
[14] T. Terasoma, An appendix to H. Endo's paper, Math. Ann., 316 (2000), 255-257.

Tatsuya Arakawa

Department of Information and Computer Engineering

Gunma NationalCollege of Technology

580 Toriba, Maebashi, Gunma, 371-8530

JAPAN

arakawa@ice.gunma-ct.ac.jp

Tadashi Ashikaga

Department of Applied Physics and Information

Faculty of Engineering, Tôhoku-Gakuin University

Chuo 1-13-1, Tagajo, Miyagi, 985-8537

JAPAN

tashikaga@tjcc.tohoku-gakuin.ac.jp 